\title{
Timpanismo ruminal crônico secundário à hipoplasia de pregas de omaso em dois bovinos
}

\author{
[Chronic ruminal bloat secondary to omasal laminae hypoplasia in two cattle] \\ R.E. Mendes $^{1}$, C.I. Schwertz ${ }^{1}$, L.G. D'Agostini ${ }^{1}$, C. Pilati $^{2}$, J.F. Edwards ${ }^{3}$ \\ ${ }^{1}$ Instituto Federal Catarinense - IFC - Campus Concórdia, SC \\ ${ }^{2}$ Universidade do Estado de Santa Catarina - UDESC - Campus Lages, SC \\ ${ }^{3}$ College of Veterinary Medicine, Texas A\&M University, College Station - Texas, EUA
}

\section{RESUMO}

Um novilho normando e outro charolês apresentando distensão abdominal, diarreia intermitente e timpanismo ruminal crônico, que iniciaram após desmame, foram enviados para necropsia. Observou-se ausência de pregas omasais associada à hipoplasia do órgão, assim como redução de tamanho das papilas ruminais e reticulares. Com base nas lesões e histórico, conclui-se que o timpanismo ruminal foi ocasionado pela falha no desenvolvimento do omaso.

Palavras-chave: bovino, timpanismo, hipoplasia, omaso

\begin{abstract}
Two emaciated juvenile steers, one Normande and one Charolaise breed with abdominal distension, intermittent diarrhea and chronic ruminal bloat that had begun at weaning were necropsied. Absence of the omasal laminae with omasal hypoplasia were found together with loss of ruminal papillae and reticular folds. Based on the lesions and history we concluded that the ruminal bloat was due to a development failure of the omasum.
\end{abstract}

Keywords: cattle, bloat, hypoplasia, omasum

\section{INTRODUÇÃO}

Timpanismo é a distensão excessiva do rúmen e retículo causada pela retenção dos gases da fermentação. É ocasionada de forma primária, por formação de espuma estável, ou secundária, devido a anormalidades funcionais e/ou físicas que interferem na eructação (Radostitis et al., 2007). Existem poucos relatos de timpanismo ruminal gasoso crônico não associado a obstruções físicas. No Estado de Santa Catarina, casos de timpanismo crônico estão frequentemente relacionados à ocorrência de carcinoma de células escamosas no cárdia devido ao consumo crônico de samambaia (Pteridium [aquilinum] arachnoideum).

Hipoplasia é descrita como o não desenvolvimento de um órgão, ou parte dele, até

Recebido em 18 de julho de 2013

Aceito em 3 de outubro de 2014

E-mail: ricardo.mendes@ifc-concordia.edu.br o seu tamanho normal. O distúrbio pode ocorrer durante o período de crescimento, pré ou pósnatal. Suas causas ainda não foram definidas, podendo ocorrer por defeitos genéticos, deficiências hormonais específicas, assim como certos agentes infecciosos ou tóxicos podem ter relação com a causa. É considerada, ainda, como resultado de falha da embriogênese em coordenar funções e estruturas durante o desenvolvimento embrionário, devido a um acidente ou ausência do código genético normal (McGavin e Zachary, 2009). Pode causar uma doença clínica, especialmente se o órgão afetado reduzir sua função ou se é imprescindível para a vida do animal, como é o caso do omaso (Radostitis et al., 2007).

A hipoplasia de omaso já foi descrita em bovinos com crescimento retardado por Nieberle e Cohrs (1970), Van Der Luer (1978), Santos (1979) e Takagi et al. (2007). 
O objetivo deste trabalho é relatar a ocorrência de timpanismo ruminal crônico secundário à hipoplasia de omaso diagnosticado em dois bovinos no Estado de Santa Catarina, Brasil.

\section{CASUÍSTICA}

Dois bovinos foram levados ao Laboratório de Patologia Animal do Centro de Ciências Agroveterinárias (CAV) da Universidade do Estado de Santa Catarina (UDESC) para necropsia nos anos de 1996 e 2002. O histórico e os sinais clínicos da doença foram obtidos junto aos veterinários responsáveis e os proprietários. Os animais foram submetidos à eutanásia e necropsia em função do prognóstico desfavorável. Fragmentos dos órgãos foram colhidos, fixados em formol a $10 \%$ e processados rotineiramente para exame histológico. Exames laboratoriais complementares não foram realizados.

Os dois animais tinham idades de 9 e 15 meses, sendo um deles da raça Normanda (bovino 1) e o outro meio sangue Charolês (bovino 2). O quadro clínico apresentado pelos bovinos iniciou-se logo após o desmame e consistia no retardo do desenvolvimento, pelos arrepiados, timpanismo recidivante e distensão abdominal bilateral, com diarreia intermitente. A auscultação revelou um murmúrio ruminal insignificante, com redução das contrações (menos de duas a cada cinco minutos). O exame fecal revelou a presença de partículas fibrosas não digeridas completamente e de tamanho maior que dois centímetros. O quadro era crônico e as condições corporais dos animais agravaramse progressivamente (Fig. 1A).

Durante a necropsia observou-se: mal estado nutricional, atrofia gelatinosa da gordura pericárdica e perirrenal, rúmen e retículo com distensão moderada e conteúdo líquido a pastoso, abomaso moderadamente distendido e com conteúdo muito grosseiro semelhante ao encontrado no rúmen. As papilas na mucosa do rúmen e retículo apresentavam-se subdesenvolvidas, com tamanho médio de 1 milímetro, e em alguns locais, ausentes. Observaram-se, ainda, no bovino 1, duas úlceras cicatrizadas de dois centímetros de diâmetro no rúmen. O omaso estava pequeno, medindo em torno de $10 \mathrm{~cm}$ de diâmetro (Fig. 1B) e, quando aberto, a mucosa apresentava apenas vestígios das folhas omasais (Fig. 1C).

$\mathrm{Na}$ histopatologia, observou-se intensa hipoplasia das pregas omasais (Fig. 1D) e do epitélio ruminal. No bovino 1 havia hiperceratinização, caracterizada por uma fina camada de material ceratinizado marrom envolvendo as pregas. No bovino 2 havia uma úlcera no epitélio omasal, com intenso infiltrado inflamatório de polimorfonucleares, formação de crostas na superfície e infiltrado multifocal moderado predominantemente linfoplasmocitário na muscular do omaso.

\section{DISCUSSÃO E CONCLUSÃO}

Doenças primárias do omaso são raras. As mais comuns são: impactação, distensão e indigestão vagal (Radostitis et al. 2002). Todas cursam com um aumento de tamanho do órgão.

O quadro clínico apresentado pelos bovinos do presente relato foi semelhante ao descrito por outros autores (Nieberle e Cohrs, 1970; Van Der Luer, 1978; Santos, 1979; Takagi et al., 2007). O estado geral caquético bastante significativo desses animais provavelmente foi resultante de uma baixa passagem do conteúdo ruminal pelo omaso, pela limitação ao consumo de matéria vegetal, e por possível comprometimento da ruminação pelo timpanismo. Assim como, também, pela baixa taxa de absorção dos alimentos ingeridos, devido ao grande número de partículas grosseiras ocasionadas pelos distúrbios de digestão ruminal. As alterações reticulorruminais poderiam ter sido causadas pelo mesmo mecanismo que originou a hipoplasia do omaso, ou mesmo por atrofia, devido à menor quantidade de alimentos que atravessavam os compartimentos gástricos.

Alhendi et al. (1996) descrevem a ocorrência de hipoplasia de retículo em uma vaca de quatro anos, que apresentava o órgão e seus pilares reduzidos de tamanho, atribuindo a sua causa a distúrbios do desenvolvimento ou a fatores nutricionais. Takagi et al. (2007) não observaram alterações histológicas na descrição de um caso de hipoplasia de omaso. Entretanto, constataram a ausência das pregas secundárias, que pode estar relacionada a diferentes graus de hipoplasia ou de sua severidade. 
Devido à grande quantidade de Pteridium [aquilinum] arachnoideum presente nas pastagens do Estado de Santa Catarina, na ocorrência de timpanismo ruminal gasoso crônico, a intoxicação por essa planta sempre deve estar listada na suspeita clínica. Contudo, em se tratando de animais jovens, essa patologia não deve ser considerada como uma causa provável para o diagnóstico clínico. E, ainda, pode ser facilmente diferenciada de uma lesão no omaso no momento da necropsia. Mas mudanças na alimentação devem ser levadas em consideração, entretanto estas acometem um número expressivo de animais do rebanho, e em geral não têm o aparecimento insidioso.

O acúmulo de gás no rúmen em animais de três a nove meses pode estar também relacionado à baixa ingestão de fibras ou ao desenvolvimento anormal do rúmen; sendo o primeiro presente em grande parte do rebanho e o segundo, diferenciado na necropsia e por exames complementares ruminais (Radostitis et al., 2007).

Obstruções esofágicas externas também devem ser consideradas, como as produzidas por compressão em casos de linfadenite e aderências por peritonite, ambas resultando em baixa contração ruminal, sendo raras nessa faixa etária.

A presença de material fibroso de dimensões maiores que o normal nas fezes de ruminantes é sugestivo de distúrbio nos pré-estômagos, já que partículas maiores tendem a permanecer no rúmen até que sejam suficientemente pequenas para passar pelo orifício retículo-omasal (Constable, 1999). Partículas maiores que cinco milímetros presentes nas fezes indicam uma rápida passagem da ingesta pelo rúmen (Constable, 1999), ou um não funcionamento do orifício reticulo-omasal (Whitlock, 1999).

O omaso localiza-se centralmente no abdômen anterior, impossibilitando a sua auscultação e palpação. Entretanto, a ausência de distúrbios de motilidade ruminal, associada a timpanismo crônico e à observação de fibras vegetais maiores de um centímetro devem ser consideradas como forte indicativo de um distúrbio omasal, especialmente em animais com emagrecimento progressivo.
A inervação do forâmen retículo-omasal é responsável, além da passagem do conteúdo pelo mesmo, também pela motilidade ruminal (Titchen, 1968), o que poderia justificar a ocorrência de timpanismo ruminal nos bovinos do presente relato; ainda que o timpanismo possa ter sido causado parcial ou exclusivamente pela retenção da ingesta na câmara ruminal, e sua consequente fermentação. Entretanto, a capacidade de eructação em animais sadios supera a máxima produção de gases pelo rúmen, mesmo nas mais altas taxas de fermentação microbiana, sendo o timpanismo causado geralmente não pela superprodução, mas sim pela falha na eliminação dos gases (Leek, 1986). Ainda, uma hipotonia ruminal, de origem reflexa pelo não funcionamento do omaso, ou por alterações na inervação como citado anteriormente, também explica o acúmulo de gás observado nestes animais.

Já observamos a ocorrência de destruição das pregas omasais (Fig. 2) em consequência da passagem de leite do rúmen ao omaso, ocasionada por debilidade geral do animal em decorrência de outras patologias ou pela falha no fechamento da goteira esofágica em caso de alimentação líquida. A fermentação láctea no rúmen leva à produção de ácido láctico, assim como à hiperqueratose ou paraqueratose da mucosa ruminal, a qual pode levar a distúrbios de motilidade ruminal com timpanismo (Amstutz, 2008). Esses casos são inicialmente acompanhados de infiltrado inflamatório na parede do órgão (mucosa e submucosa, podendo alcançar a muscular e a serosa, dependendo da sua gravidade). Entretanto, posteriormente à resolução e cicatrização, as células inflamatórias tendem a desaparecer, e pode observar-se fibrose do órgão afetado.

A presença de úlceras na parede do rúmen do bovino 1 é compatível com o quadro clínico de fermentação láctea no rúmen; contudo, o aparecimento gradual dos sinais clínicos somente após o desmame apoia o diagnóstico de hipoplasia de omaso, já que o mesmo terá maior importância funcional quando os animais passarem a ingerir maior quantidade de matéria vegetal. Como a formação da goteira esofágica não é dependente da inervação ou musculatura do omaso (Blowey, 1996), a hipofuncionalidade do órgão desde o nascimento não acarretará distúrbios gástricos evidentes enquanto o animal 
Timpanismo ruminal crônico...

for lactante. Entretanto, os bovinos necropsiados neste estudo não foram alimentados em nenhum momento com rações à base de grãos, leite ou seus sucedâneos, não sendo possível encontrar uma origem para a possível acidose nos préestômagos além da ocasionada por distúrbios de fechamento da goteira esofágica.
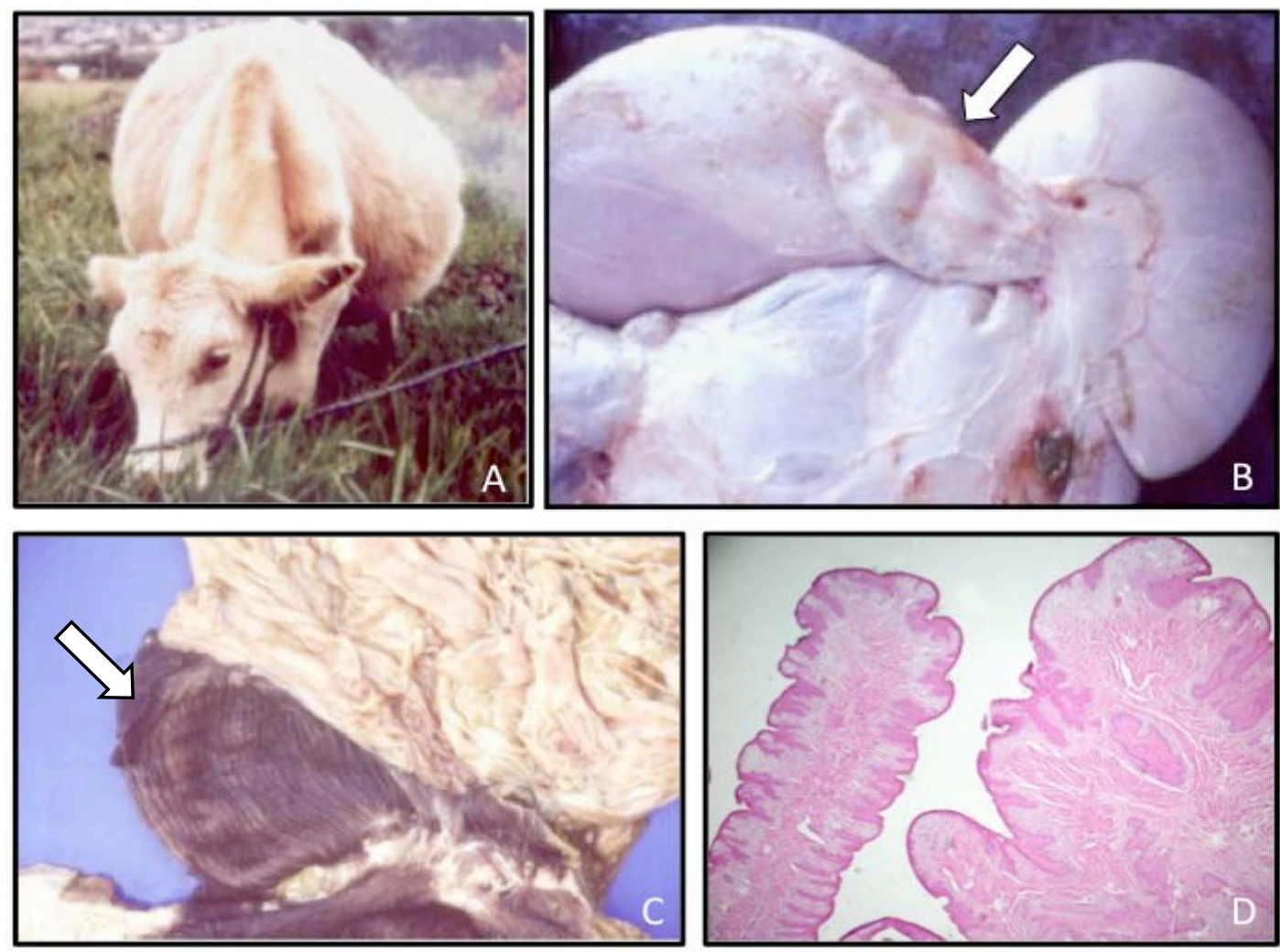

Figura 1. Bovino 2. A. Distensão abdominal e estado nutricional ruim. B. Reduzido tamanho do omaso (seta). C. Ausência das pregas do omaso aberto (seta). D. Hipoplasia das pregas do omaso. HE, obj.10x.

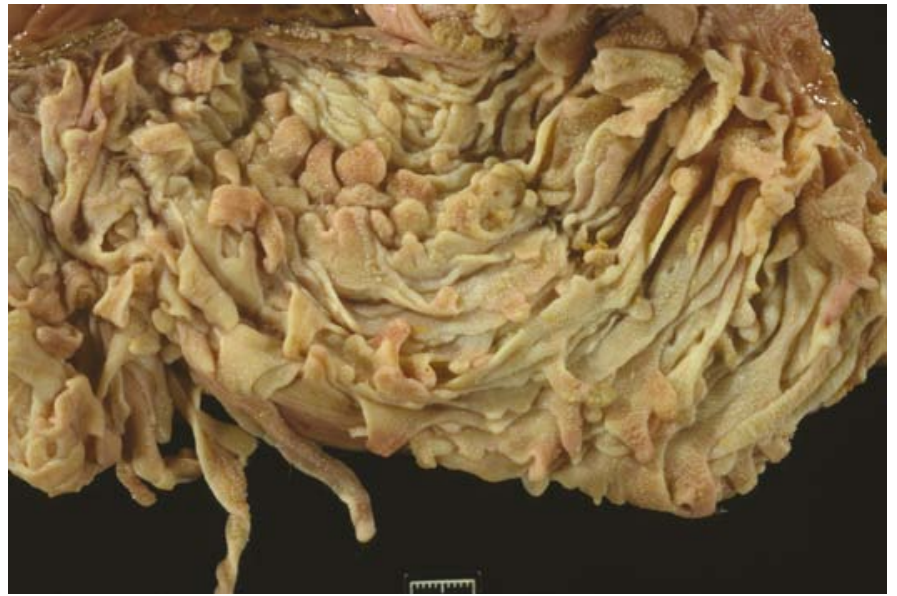

Figura 2. Bovino. Omaso aberto. Pregas irregulares e de reduzido tamanho devido à acidose ruminal por excessivo consumo de sucedâneo de leite. 
Os dois bovinos necropsiados eram provenientes de propriedades geograficamente distantes, não apresentando parentesco algum, descartando-se a consanguinidade como fator importante ao desenvolvimento da patologia.

Com base no exposto, conclui-se que os casos de emagrecimento progressivo e timpanismo crônico aqui descritos foram causados pela hipoplasia das pregas do omaso, que provavelmente era de origem congênita primária.

\section{AGRADECIMENTO}

O trabalho foi publicado com apoio financeiro do Instituto Federal Catarinense (IFC) através do edital 203/2013 PROPI.

\section{REFERÊNCIAS}

ALHENDI, A.B.; GAMEEL, A.A.; RAMADAN, R.O. Possible hypoplasia of the reticulum in a cow. Can. Vet. J., Ottawa, v.37, p.442-443, 1996.

AMSTUTZ, H.E. Manual Merk de Veterinária. 9.ed., São Paulo: ROCA, 2008. 2336p.

CONSTABLE, P.D. Function and dysfunction of the ruminant forestomach. In: HOWARD, J.L.; SMITH, R.A. (Eds). Current Veterinary Therapy Food Animal Practice. 4.ed. Pennsylvania: SAUNDERS, 1999. p.502-507.

BLOWEY, R. Calf feeding practices in relation to calf health. Agri-Practice, v.17, p.20-22, 1996.
LEEK, B.F. Clinical diseases of the rumen: A physiologist's view. Vet. Rec., v.113, p.10-14, 1983.

MCGAVIN, M.D.; ZACHARY, J.F. Bases da Patologia em Veterinária. 4.ed. São Paulo: ELSEVIER, 2009. 1504p.

NIEBERLE, K.; COHRS, P. Anatomia Patológica Especial dos Animais Domésticos. 5.ed. Lisboa: FUNDAÇÃO CALOUSTE GULBENKIAN, 1970. 432p.

RADOSTITIS, O.M.; GAY, C.C.; HINCHCLIFF, K.W. et al. Veterinary Medicine. 10.ed. St. Louis: SAUNDERS, 2009. 2065p.

SANTOS, A. Patologia especial dos animais domésticos. 2.ed. Rio de Janeiro: INTERAMERICANA, 1979. 394p.

TAKAGI, M.; MUKAI, S.; FUSHIMI, Y. et al. Severe Hypoplasia of the Omasal Laminae in a Japanese Black Steer with Chronic Bloat - A Case Report. J. Vet. Med. Sc., v.69, p.1281-1286, 2007.

TITCHEN, D.A. Section 6: The Gastrointestinal System. In: CODE, C.F. (Ed). Handbook of Physiology. Washington: AMERICAN PHYSIOLOGICAL SOCIETY, 1968. p.27052725.

VAN DER LUER, R.J. Malformation of laminae omasi in cattle. Tijdschr Diergeneeskd, v.108, p.956-563, 1978.

WHITLOCK, R. Vagal indigestion. In: HOWARD, J.L.; SMITH, R.A. (Eds). Current Veterinary Therapy: Food animal practice. 4.ed. Pennsylvania: SAUNDERS, 1999. p.517-522. 